\title{
Asymptotic behaviour of solutions to the Korteweg-deVries-Burgers system
}

\author{
by \\ M. E. SCHONBEK * and S. V. RAJOPADHYE \\ Department of Mathematics, \\ University of California at Santa Cruz
}

\begin{abstract}
We consider the large time behaviour of solutions ot the Korteweg-deVries-Burgers system of equations to obtain lower and upper bounds for the rates of decay of the solution. These decay rates extend the work of Amick et al. [1], where the scalar case was considered and that of Zhang Linghai [8]. An important tool in the analysis is the so called Fourier Splitting method developed by M. E. Schonbek for obtaining algebraic upper bounds for the solution to the system of parabolic conservation laws. This tool was later used to establish algebraic upper and lower bounds for the Navier Stokes and Magneto Hydrodynamic equations. The lower bounds show that in the far field the behaviour of the solutions to the KdVB system and those of the heat system are very different. This behaviour is believed to be due to the nonlinearity and not to the dispersive nature of the equation, since such behaviour is also present in non-dispersive systems like the Navier-Stokes and the Magneto-Hydrodynamic equations.
\end{abstract}

Key words: Korteweg-deVries-Burgers equation, decay of solutions.

RÉSUMÉ. - Dans ce travail nous obtenons des bornes supérieures et inférieures pour le taux de décroissance des solutions du système de Korteweg-deVries-Burger. Ce travail prolonge celui de Amick et al. [1] dans lequel le cas scalaire est considéré, ainsi que le travail de Zhang Linghai. La technique qui est fondamentale pour les résultats que nous obtenons est le «Fourier splitting ». Cette méthode a été développée par

AMS (MOS) Classification: 35, 76 B

* The work of this author was partially supported by NSF Grant No. DMS 9307497.

Annales de l'Institut Henri Poincaré - Analyse non linéaire - 0294-1449 
M. E. Schonbek pour obtenir des bornes supérieures algébriques pour un système de lois paraboliques de conservation. Elle a été utilisée ensuite pour établir les bornes algébriques supérieures et inférieures pour les solutions des équations de Navier-Stokes et de Magnéto-Hydrodynamique. La borne inférieure montre que pour $t$ tendant vers l'infini, le comportement des solutions pour les systèmes de Korteweg-deVries-Burger et le comportement des solutions de l'équation de la chaleur sont différents : ce comportement proviendrait des termes non linéaires et non de la nature dispersive du système car il est aussi présent dans des systèmes non dispersifs comme les équations de Navier-Stokes et les équations de Magnéto-Hydrodynamique.

\section{INTRODUCTION}

In this work we study the asymptotic behaviour of solutions to the Korteweg-deVries-Burgers system (henceforth referred to as the KdVB system), in $n$-space dimensions. This equation can be expressed in the form

$$
\begin{gathered}
U_{t}+\sum_{i=1}^{n} \frac{\partial}{\partial x_{i}} \nabla \Phi(U)+\sum_{i=1}^{n} \frac{\partial}{\partial x_{i}} \sum_{i=1}^{n} \frac{\partial}{\partial x_{i}^{k}} U \\
=\alpha \Delta U+\beta \sum_{i=1}^{n} \frac{\partial}{\partial x_{i}} U \\
U(x, 0)=U_{0},
\end{gathered}
$$

where $x=\left(x_{1}, x_{2}, \ldots, x_{n}\right) \in \mathbb{R}^{n}, U(x, t)=\left(U_{1}(x, t), \ldots, U_{n}(x, t)\right)$ is the $n$-dimensional vector valued function, $\Phi(U)$ is a scalar function of the vector variable $U$ which satisfies certain growth conditions which will be specified below. The gradient operator with respect to $U$ is denoted by the symbol "grad", $\delta_{k}=\sum_{i=1}^{n} \frac{\partial^{k}}{\partial x_{i}^{k}}, \delta=\delta_{1}$ and $p, n$ are integers greater than or equal to 1 and $\alpha>0, \beta$ are constants and $2 p>n>1$. Note that we can without loss of generality, choose $\beta$ equal to zero. This is possible by a change of coordinates, from a stationary to a moving frame of reference, which enables us to absorb the term $\beta \delta U$ into the term $U_{t}$. Moreover, we can for simplicity assume $\alpha=1$. Thus we consider the system 1 which 
can be rewritten as,

$$
\begin{gathered}
U_{t}+\delta \operatorname{grad} \Phi(U)+\delta \delta_{2 p} U=\delta_{2} U, \\
U(x, 0)=U_{0},
\end{gathered}
$$

In one dimension, the equation reduces to the generalized KdVB equation. When the nonlinearity is given by $U U_{x}$, Amick et al. [1] have obtained sharp rates of decay for the solutions to the KdVB equation. For the scalar case, Zhang Linghai has also obtained decay rates in $L_{2} \cap L_{\infty}$ for a class of equations more general than the KdVB equation; e.g. the Benjamin-Ono-Burgers equation.

In higher dimensions, existence results have been obtained by Zhang [8] following the lines of the proof of [7] for the generalized K-dV equation. Moreover, he obtains rates of decay for the $L_{2^{-}}$and $L_{\infty}$-norms of the solution as $t \rightarrow \infty$.

In this work, we first obtain decay rates on the $H^{m}$-norm and by an application of standard Sobolev inequalities obtain the $L_{\infty}$-decay rate. In addition, lower bounds on the energy decay rates of the solutions are also obtained. It is shown that for a certain class of the initial data, the solution $U(x, t)$ to the $\mathrm{KdVB}$ system admits an algebraic lower bound on the energy decay. Two distinct cases have to be considered. First, when the average of the initial data is non-zero and second, when the average equals zero. In the first case, it can be shown that

$$
|U(\cdot, t)|_{L_{2}}^{2} \geq C(t+1)^{-\alpha}
$$

where $\alpha=\frac{n}{2}$. In the second case, if the average is zero, that is the Fourier transform at the origin is zero, two cases are considered. If the zero is of order one, and the data $U_{0}$ lies in $L_{1} \cap H^{p+1}$ as well as in suitably weighted $L_{s}$ spaces, with $s=1,2$; then the lower bound is of the form

$$
|U(\cdot, t)|_{L_{2}}^{2} \geq C(t+1)^{-\alpha_{1}}
$$

where $\alpha_{1}=\frac{n}{2}+1$ and $C$ depends on the initial data and certain initial parameters. If the zero is of order greater than one, the data lies outside a set of equidistributed energy and the nonlinear function $\Phi$ lies in a large class of polynomias, then the lower bound is again of order $\alpha_{1}$. It is this second case which is the more subtle one and which shows the differences between the behaviour of solutions to the KdVB and heat systems in the far field. This explains our interest in studying the lower bound of rates of decay.

This difference in the behaviour of the KdVB and the heat system shows that the nonlinear term produces some mixing of the Fourier modes creating 
long waves even when the initial data is highly oscillaroty. More precisely if the initial data for the heat system is highly oscillaroty the solution will have an exponential rate of decay. Moreover, depending on the data chosen for the heat equation the decay in the energy norm can vary from order $(t+1)^{-\frac{n}{4}}$ (when no oscillations are present at the origin in Fourier space), to all possible algebraic orders up to exponential decay, depending on how oscillatory the data is. We restrict attention to the case where the nonlinearity is polynomial. By obtaining an algebraic lower bound for the solutions of the KdVB system we show that long wages are produced which slow down the decay. We believe that his phenomenon is due to the nonlinear term and not due to the dispersive term, since this behaviour is also present in non-dispersive systems like the Navier-Stokes and the Magneto-Hydrodynamic equations [4], [5].

The method used here is based on the Fourier Splitting technique, developed by Schonbek [2], to obtain upper bounds for solutions to the Navier-Stokes equation and parabolic conservation laws as well as for obtaining lower bounds on the solution to the Navier-Stokes and Magneto-Hydrodynamics equations.

The paper is organized as follows. In section 2, we briefly review the notational conventions. Section 3 deals with obtaining the $H_{m}$ decay rates of the solution of the KdVB system. By a simple corollary using Sobolev inequalities the $L_{\infty}$ decay rate follows. This rate coincides with the one obtained by Zhang [8]. For the sake of completeness, we present some results for the heat equation in section 4 . In section 5 , upper bounds for the difference of the solution to the heat system and the KdVB system are obtained, when both solutions correspond to the same initial data. Finally the lower bounds for the solution to the KdVB system are derived in section 6 .

\section{NOTATION}

The notation that we use is mostly standard. For the sake of completeness, it is briefly reviewed here. For $1 \leq p \leq \infty$ we denote by $L_{p}=L_{p}\left(\mathbb{R}^{n}\right)$ the Banach space of measurable real-valued functions defined on $\mathbb{R}^{n}$ which are $p$ th power Lebesgue integrable. (essentially bounded in the case $p=\infty$ ). The usual norm on the space is denoted by $|\cdot|_{p}$. For non-negative integers $s, H^{s}=H^{s}\left(\mathbb{R}^{n}\right)$ is the Sobolev space of functions in $L_{2}$ whose generalized derivatives up to order $s$ also belong to $L_{2}$. The space is equipped with 
the norm,

$$
\|f\|_{s}^{2}=\sum_{j=0}^{s}\left|f^{(j)}(\cdot)\right|_{2}, \quad \text { where } f^{(j)}=\sum_{|\alpha|=j} D^{\alpha} f .
$$

Of course $H^{0}=L_{2}$ and the $L_{2}$-norm $|\cdot|_{2}=\|\cdot\|_{0}$ will be denoted by the symbol $|\cdot|_{2}$. We also define a norm on $H^{s}$, equivalent to the usual one by,

$$
\|U(\cdot, t)\|_{s}=\sum_{r=0}^{s} k_{r, s} \sum_{|\beta|=r} \int_{\mathbf{R}^{n}}\left|D^{\beta} U(x, t)\right|^{2} d x,
$$

where the $k_{r, s}$ 's are positive constants that depend only on $r, s$ and $\beta$.

The space $C^{r}\left(\mathbb{R}^{n}, \mathbb{R}\right)$ is the space of all continuous functions from $\mathbb{R}^{n} \rightarrow \mathbb{R}$ which are $r$ times differentiable with continuous derivatives. In addition we also define some weighted spaces as follows.

$$
W_{1}=\left\{U: \int_{\mathbf{R}^{n}}|x|^{2}|U| d x<\infty\right\}
$$

and

$$
W_{2}=\left\{U: \int_{\mathbf{R}^{n}}|x||U|^{2} d x<\infty\right\}
$$

The spaces are equipped with the norms $|U|_{W_{1}}=\int_{\mathbf{R}^{n}}|x|^{2}|U| d x$; $|U|_{W_{2}}=\left(\int_{\mathbf{R}^{n}}|x||U|^{2} d x\right)^{\frac{1}{2}}$ respectively.

The Fourier transform of a function $f(x)$ is denoted by $\mathcal{F}(f(\xi))$ and is given by

$$
\mathcal{F}(f(\xi))=\int_{\mathbf{R}^{n}} e^{-2 \pi i \xi \cdot x} f(x) d x .
$$

The notation $D^{\beta}$ denotes the derivative of order $\beta$ where $\beta$ is a multi-index i.e., if

$$
\beta=\left(\beta_{1}, \beta_{2} \ldots, \beta_{n}\right)
$$

then

$$
D^{\beta}=\partial_{x_{1}}^{\beta_{1}} \partial_{x_{2}}^{\beta_{2}} \ldots \partial_{x_{n}}^{\beta_{n}}
$$

Vol. 12, $\mathrm{n}^{\circ} 4-1995$. 


\section{BOUNDS ON THE $H^{m}$ AND $L_{\infty}$-NORM OF THE SOLUTION}

Equation (1) has been studied by Zhang [8] to obtain results on the existence of weak solutions under the hypothesis that the nonlinearity (defined by $\Phi$ ) satisfies the following growth conditions.

$$
\left\{\begin{array}{l}
\Phi(U) \leq C|U|^{\frac{4 p}{n}+2-q}+C|U|^{2}, \\
0<q<1, \\
\left|\frac{\partial^{2} \Phi(U)}{\partial U_{i} \partial U_{j}}\right| \leq C|U|^{\frac{4 p}{n}}+C, \\
\text { for all } U \in \mathbb{R}^{n}, \quad i, j=1,2, \ldots, n \\
\left|\frac{\partial^{2} \Phi(U)}{\partial U_{i} \partial U_{j}}\right| \leq C|U|^{\frac{4 p}{n}}+1, \\
\text { for }|U| \text { small, } i, j=1,2, \ldots, n .
\end{array}\right.
$$

In addition, Zhang uses estimates on the $L_{2}$ - and the $H^{s}$-norms of the solution to show that if $U_{0} \in L_{1} \cap H^{p+1}$ the weak solution is a strong solution. In the present work, we further restrict $\Phi$ to satisfy the condition

$$
\Phi(U)=\sum_{i, k} m_{i k} U_{i}^{k_{i}}
$$

where $m_{i}$ are real scalars and $3<k_{i} \leq \frac{4 p}{n}+2-q$, with $p>1$. The case when $k_{i}=0,1,2$ corresponds to the linear equation and will not be considered for the lower bounds. Hence it also follows that,

$$
\left\{\begin{array}{l}
|\Phi(U)| \leq C|U|^{\frac{4 p}{n}+2-q}, \quad 0<q<1 \\
\left|\frac{\partial^{2} \Phi(U)}{\partial U_{i} \partial U_{j}}\right| \leq C|U|^{\frac{4 p}{n}} \\
\left|\frac{\partial^{2} \Phi(U)}{\partial U_{i}}\right| \leq C|U|^{\frac{4 p}{n}+1}
\end{array}\right.
$$

for all $U \in \mathbb{R}^{n}$ and $i, j=1,2, \ldots, n$. Zhang has shown (cf. Theorem 6 in [8]) that if $U_{0} \in L_{1} \cap H^{p+1} ; \Phi(U) \in C^{3}\left(\mathbb{R}^{n}\right)$ and satisfies (5), the following decay properties hold for the solution to the KdVB system:

$$
\left\{\begin{array}{l}
|U(t)|_{2} \leq C(t+1)^{-\frac{n}{4}} \\
|U(t)|_{\infty} \leq C(t+1)^{-\frac{n}{4}}
\end{array}\right.
$$


Here we first obtain the decay rate for the $H^{s}$-norm of the solution. Then, making use of standard Sobolev inequalities, the decay rate obtained in [8] for the $L_{\infty}$-norm follows.

THEOREM 3.1. - If $\Phi(U) \in C^{m+1}\left(\mathbb{R}^{n}, \mathbb{R}\right)$ and satisfies equation (5) with $U_{0} \in L_{1} \cap H^{p+1}\left(\mathbb{R}^{n}\right)$, then the solution $U$ to the $K d V B$ system with initial data $U_{0}$ is such that,

$$
\sum_{|\alpha| \leq m} \int_{\mathbb{R}^{n}}\left|D^{\alpha} U\right|^{2} d x \leq C(t+1)^{-\frac{n}{2}} .
$$

Moreover, if $m>\left[\frac{n}{2}\right]$ then,

$$
\sum_{|\alpha| \leq m-\left[\frac{n}{2}\right]}\left|D^{\alpha} U\right|_{\infty} \leq C(t+1)^{-\frac{n}{4}},
$$

where the constant $C$ depends only on $\Phi, n, m$ and $U_{0}$.

Proof. - To obtain decay rates for the $H^{s}$-norm of the solution of the KdVB system, we use induction. We begin by giving an equivalent definition for the $H^{s}$-norm. Define,

$$
\||U(t)|\|_{s}^{2}:=\sum_{r=0}^{s} k_{r, s} \sum_{|\boldsymbol{\beta}|=r} \int_{\mathbf{R}^{n}}\left|D^{\beta} U(x, t)\right|^{2} d x,
$$

where the $k_{r, s}$ 's are positive constants depending on $r, s, \beta$ and $|U|_{\infty}$ which will be determined below. We will show by induction that

$$
\frac{d}{d t}\||U|\|_{s}^{2} \leq-C \sum_{r=1}^{s+1} k_{r, s} \sum_{|\beta|=r} \int\left|D^{\beta} U\right|^{2} d x
$$

and

$$
\|\| U \|_{s}^{2} \leq C(t+1)^{-\frac{n}{2}} .
$$

For $|s|=0$ inequality (8) follows by multiplying the equation by $U$ and integrating in space and inequality (9) reduces with $k_{0,0}=1$ to

$$
\int_{\mathbb{R}^{n}}|U(t)|^{2} d x \leq C(t+1)^{-\frac{n}{2}}
$$


a result obtained in [8]. For the sake of completeness, we give a sketch of the proof here. Multiply equation (2) by $U$ and integrate over space to get,

$$
\begin{aligned}
\frac{1}{2} \frac{d}{d t} \int|U(x, t)|^{2} d x & +\int U \delta \Phi(U) d x \\
& +\int U \delta \delta_{2 p} U d x=\int U \delta_{2} U d x
\end{aligned}
$$

On simplification, we have,

$$
\frac{d}{d t} \int|U(x, t)|^{2} d x=-2 \int|\nabla U|^{2} d x .
$$

By Plancherel's theorem the equality can be rewritten as

$$
\frac{d}{d t} \int|\hat{U}|^{2} d \xi=-2 \int|\xi|^{2}|\hat{U}|^{2} d \xi
$$

Using the Fourier Splitting technique introduced by Schonbek [2], the Fourier space is divided into two time dependent disjoint sets $A(t)$ and $A(t)^{c}$, where

$$
A(t):=\left\{\xi:|\xi| \leq\left[\frac{n}{2(t+1)}\right]^{\frac{1}{2}}\right\}
$$

Inequality (12) can then be rewritten as,

$$
\begin{aligned}
\frac{d}{d t} & \int_{\mathbf{R}^{n}}|U|^{2} d \xi \\
& \leq-2 \int_{A(t)}|\xi|^{2}|\hat{U}|^{2} d \xi-2 \int_{A(t)^{c}}|\xi|^{2}|\hat{U}|^{2} d \xi \\
& \leq-\frac{n}{t+1} \int_{A^{c}(t)}|\hat{U}|^{2} d \xi \\
& \leq-\frac{n}{t+1} \int_{\mathbf{R}^{n}}|\hat{U}|^{2} d \xi+\frac{n}{t+1} \int_{A(t)}|\hat{U}|^{2} d \xi
\end{aligned}
$$

Hence it follows that,

$$
\frac{d}{d t}\left[(t+1)^{n} \int_{\mathbb{R}^{n}}|\hat{U}|^{2} d \xi\right] \leq n(t+1)^{n-1} \int_{A(t)}|\hat{U}|^{2} d \xi
$$

Moreover, it has been shown in [8] that if $U_{0} \in L_{1} \cap H^{p+1}$, then

$$
|\hat{U}(\cdot, t)|_{\infty} \leq\left|U_{0}\right|_{1}+\left\|U_{0}\right\|_{p}^{2}
$$


Equation (15) together with this time independent bound for the Fourier transform yields,

$$
\left\{\begin{aligned}
\frac{d}{d t} & {\left[(t+1)^{n} \int_{\mathbb{R}^{n}}|\hat{U}|^{2} d \xi\right] } \\
& \leq n(t+1)^{n-1}\left(\left|U_{0}\right|_{1}^{2}+\left\|U_{0}\right\|_{p}^{2}\right)^{2} \int_{A(t)} d \xi \\
& \leq\left(\left|U_{0}\right|_{1}+\left\|U_{0}\right\|_{p}^{2}\right)^{2} n(t+1)^{n-1} \omega_{n}\left(\frac{n}{2(t+1)}\right)^{\frac{n}{2}}
\end{aligned}\right.
$$

where $\omega_{n}$ denotes the volume of the $n$-dimensional unit sphere. Integrating (17) over the interval $[0, t]$ inequality (10) follows. This proves the estimate in the case $|s|=0$.

Define,

$$
\|U(t)\|_{s}^{2}:=\sum_{r=0}^{s} k_{r, s} \sum_{|\beta|=r} \int_{\mathbb{R}^{n}}\left|D^{\beta} U(x, t)\right|^{2} d x .
$$

By the induction hypothesis, it follows that,

$$
\frac{d}{d t}\|U\|_{s-1}^{2} \leq-C \sum_{r=0}^{s} k_{r, s-1} \sum_{|\beta|=r} \int\left|D^{\beta} U\right|^{2} d x
$$

and

$$
\|U\|_{s-1}^{2} \leq C(t+1)^{-\frac{n}{2}}
$$

Hence, for an appropriate choice of constants $k_{r, s}$, it is necessary to show that

$$
\|U(t)\|_{s}^{2} \leq C(t+1)^{-\frac{n}{2}}
$$

which would prove the desired result. Multiply equation (2) by $D^{2 \beta} U$ and integrate over space. This yields,

(19) $(-1)^{\beta} \frac{1}{2} \frac{d}{d t} \int_{\mathbb{R}^{n}}\left|D^{\beta} U\right|^{2} d x+\int_{\mathbb{R}^{n}} D^{2 \beta} U \delta \operatorname{grad} \Phi(U) d x$

$$
+\int_{\mathbb{R}^{n}} D^{2 \beta} U \delta \delta_{2 p} U d x=(-1)^{\beta+1} \sum_{j=1}^{n} \int_{\mathbb{R}^{n}}\left|D^{\beta+e_{j}} U\right|^{2} d x
$$


Note that the third term on the left-hand side integrates to zero. Using the definition of $\Phi$ given by (4) and hence determining the growth condition on $\operatorname{grad} \Phi$, the second term on the right-hand side of (19) can be bounded above by

$$
C \int_{\mathbb{R}^{n}} \delta\left(\sum U_{i}^{k_{i}-1}\right) D^{2 \beta} U d x
$$

where $k_{i} \leq \frac{4 p}{n}+2-q$. Thus it follows that $2<k=k_{i}-1 \leq \frac{4 p}{n}+1$. Combining this bound with equation (19) yields,

$$
\begin{aligned}
\frac{d}{d t} & \int_{\mathbb{R}^{n}}\left|D^{\beta} U\right|^{2} d x \\
\leq & -2 \sum_{j=1}^{n} \int_{\mathbb{R}^{n}}\left|D^{\beta+\varepsilon_{j}} U\right|^{2} d x \\
& +C \sum_{j=1}^{n} \int_{\mathbb{R}^{n}} D^{\beta+\varepsilon_{j}} U D^{\beta} U^{k} d x \\
\leq & -2 \sum_{j=1}^{n} \int_{\mathbb{R}^{n}}\left|D^{\beta+\varepsilon_{j}} U\right|^{2} d x \\
& +C\left(\sum_{j=1}^{n} \frac{1}{C} \int_{\mathbb{R}^{n}}\left|D^{\beta+\varepsilon_{j}} U\right|^{2} d x\right. \\
& \left.\quad+\sum_{j=1}^{n} \frac{C}{4} \int_{\mathbb{R}^{n}}\left|D^{\beta} U^{k}\right|^{2} d x\right) \\
\leq & -\sum_{j=1}^{n} \int_{\mathbb{R}^{n}}\left|D^{\beta+\varepsilon_{j}} U\right|^{2} d x+\frac{C^{2}}{4} \int_{\mathbb{R}^{n}}\left|D^{\beta} U^{k}\right|^{2} d x \\
\leq & -\sum_{j=1}^{n} \int_{\mathbb{R}^{n}}\left|D^{\beta+\varepsilon_{j}} U\right|^{2} d x+\frac{C^{2}}{4} C_{0} \int_{\mathbb{R}^{n}}\left|D^{\beta} U\right|^{2} d x \\
& +\sum_{|\alpha| \leq \beta} C_{\alpha, \beta} \int_{\mathbb{R}^{n}}\left|D^{\alpha} U\right|^{2} d x .
\end{aligned}
$$

In the second step we have made use of Schwartz's inequality, and in the last step we use the fact that if $s>k$ we have

$$
D^{s} U^{k}=\sum_{j=1}^{s} C_{k, s} U^{k-s-j}\left(\sum_{\alpha r=j}\left(D^{\alpha} U\right)^{r}\right), \quad r \text { integer }
$$


and if $s \leq k$

$$
D^{s} U^{k}=\sum_{\alpha_{1}+\ldots+\alpha_{k}=s} D^{\alpha_{1}} U \ldots D^{\alpha_{k}} U
$$

and that $j \leq s$ and $\alpha, r \leq s$. In addition, by the induction hypothesis, since derivatives up to order $s-1$ are bounded in $L_{2}$, the last inequality in (20) follows by using a simple interpolation inequality and $C_{0}$ denotes a constant depending on $\Phi$ and the $L_{\infty}$-norms of $U$ and its derivatives up to order $\beta-\left[\frac{n}{2}\right]$. Hence inequality (20) yields,

$$
\begin{aligned}
\frac{d}{d t} \int_{\mathbf{R}^{n}}\left|D^{\beta} U\right|^{2} d x \leq & -\sum_{j=1}^{n} \int_{\mathbb{R}^{n}}\left|D^{\beta+\varepsilon_{j}} U\right|^{2} d x \\
& +C_{1} \int_{\mathbf{R}^{n}}\left|D^{\beta} U\right|^{2} d x \\
& +\sum_{|\alpha| \leq \beta} C_{\alpha, \beta} \int_{\mathbf{R}^{n}}\left|D^{\alpha} U\right|^{2} d x .
\end{aligned}
$$

Summing over all $\beta$ such that $|\beta|=s$ leads to

$$
\begin{aligned}
\frac{d}{d t} & {\left[\int_{\mathbb{R}^{n}} \sum_{|\beta|=s}\left|D^{\beta} U\right|^{2} d x\right] } \\
\leq & C_{s}\left(C_{1} \int_{\mathbb{R}^{n}} \sum_{|\beta|=s}\left|D^{\beta} U\right|^{2} d x\right) \\
& -\sum_{|\beta|=s+1}\left(\int_{\mathbb{R}^{n}}\left|D^{\beta} U\right|^{2} d x\right) .
\end{aligned}
$$

By the inductive hypothesis,

$$
\frac{d}{d t}\left[\|\mid U(t)\|_{s-1}^{2}\right] \leq-C \sum_{r=1}^{s} k_{r, s} \sum_{|\beta|=r} \int_{\mathbb{R}^{n}}\left|D^{\beta} U\right|^{2} d x
$$


Let $m_{s-1}=C k_{r, s-1} r \leq s-1$. Choose $k_{s+1}=m_{s-1}\left(2 C_{s} C_{1}\right)^{-1}$. Adding together inequalities (23) and (22) times $k_{s+1}$ we get,

$$
\begin{aligned}
\frac{d}{d t}\|U\| \|_{s}^{2} \leq & -C \sum_{r=0}^{s} k_{r} \sum_{|\beta|=s} \int_{\mathbb{R}^{n}}\left|D^{\beta} U\right|^{2} d x \\
& +C \max _{r \leq s} k_{r, s-1} \int_{\mathbb{R}^{n}} \sum_{|\beta|=s}\left|D^{\beta} U\right|^{2} d x \\
& -R_{s+1} \sum_{|\beta|=s+1} \int_{\mathbb{R}^{n}}\left|D^{\beta} U\right|^{2} d x \\
\leq & -K_{s}\left(\sum_{r=1}^{s+1} k_{r} \sum_{|\beta|=r} \int_{\mathbb{R}^{n}}\left|D^{\beta} U\right|^{2} d x\right),
\end{aligned}
$$

where $K_{s}=\min \left\{1, K_{s-1}\right\}$, and $R_{s+1}$ is proportional to $m_{s-1}$. Let $k_{r, s}=k_{r, s-1} / 2, k_{s, s}=k_{s+1}$ and $k_{s+1, s}=k_{s, s}$. This establishes the inequality

$$
\frac{d}{d t}\|U(t)\|_{s}^{2} \leq-C \sum_{r=1}^{s+1} k_{r, s} \sum_{|\boldsymbol{\beta}|=r} \int_{\mathbb{R}^{n}}\left|D^{\beta} U\right|^{2} d x
$$

If $|\beta|=0(24)$ reduces to obtaining the $L_{2}$-rate of decay for $U$, which has been established in (10). Suppose that the decay rate has been obtained for $\beta$ with $0 \leq|\beta|<s$. Let $|\beta|=s$. Taking the Fourier transform of inequality (24) we obtain,

$$
\begin{aligned}
\frac{d}{d t}\|\hat{U}\|_{s}^{2}= & \frac{d}{d t} \sum_{r=0}^{s} k_{r, s} \sum_{|\beta|=r} \int_{\mathbf{R}^{n}}\left|\widehat{D^{\beta} U}\right|^{2} d \xi \\
& \leq-C \sum_{r=1}^{s+1} k_{r, s} \sum_{|\beta|=r} \int_{\mathbf{R}^{n}}\left|\widehat{D^{\beta} U}\right|^{2} d \xi \\
& =-C \sum_{r=1}^{s} k_{r, s} \sum_{|\beta|=r} \int_{\mathbf{R}^{n}}|\xi|^{2}\left|\widehat{D^{\beta} U}\right|^{2} d \xi .
\end{aligned}
$$


Repeating the same argument in (25) as that used in obtaining (17), we have,

$$
\begin{aligned}
\frac{d}{d t} & {\left[(t+1)^{n}\|\mid U(t)\|_{s}\right] } \\
& =\frac{d}{d t}\left[(t+1)^{n} \sum_{r=0}^{s} k_{r, s} \sum_{|\beta|=r} \int_{\mathbb{R}^{n}}\left|\widehat{D^{\beta} U}\right|^{2} d \xi\right] \\
& \leq K_{s}(t+1)^{n-1} \sum_{r=0}^{s} k_{r, s} \sum_{|\beta|=r} \int_{A(t)}\left|\widehat{D^{\beta} U}\right|^{2} d \xi
\end{aligned}
$$

where $A(t)$ is as defined earlier in (13). Hence, if $K_{s}=\max k_{r, s}$

$$
\text { (26) } \begin{aligned}
\frac{d}{d t}\left[(t+1)^{n}\|\hat{U} \mid\|_{s}^{2}\right] \leq & C(t+1)^{n-1} K_{s} \sum_{|\beta|=r} \int_{A(t)}\left|\widehat{D^{\beta} U}\right|^{2} d \xi \\
\leq & C(t+1)^{n-1} K_{s} \sum_{|\beta|<s} \int_{\mathbf{R}^{n}}\left|\widehat{D^{\beta} U}\right|^{2} d \xi \\
& +C(t+1)^{n-1} \int_{A(t)}|\xi|^{2}\left|\widehat{D^{s-1}} U\right|^{2} \\
\leq & C(t+1)^{n-1} K_{s} \sum_{|\beta|<s} \int_{\mathbf{R}^{n}}\left|\widehat{D^{\beta} U}\right|^{2} d \xi \\
& +C(t+1)^{n-2} \int_{\mathbf{R}^{n}}\left|\widehat{D^{s-1}} U\right|^{2} d \xi .
\end{aligned}
$$

By the inductive hypothesis for $|\beta|<s$,

$$
\int_{\mathrm{R}^{n}}\left|\widehat{D^{\beta} U}\right|^{2} d \xi \leq C(t+1)^{-\frac{n}{2}}
$$

Using this in (26) we obtain,

$$
\frac{d}{d t}\left[(t+1)^{n}\|\hat{U}\|_{s}^{2}\right] \leq C(t+1)^{\frac{n}{2}-1}
$$

Integrating this last expression over the interval $[0, t]$ yields

$$
(t+1)^{n}\|U(t)\|_{s}^{2} \leq C(t+1)^{\frac{n}{2}}+\|U(0)\|_{s}^{2},
$$

Vol. $12, \mathrm{n}^{\circ} 4-1995$. 
which proves the claim. The bounds for the $L_{\infty}$-norm of $U$ follow from inequality (27) and Sobolev's inequality. Hence we obtain,

$$
\sum_{|\beta|=j}\left|D^{\beta} U\right|_{\infty} \leq C\|U\|_{m}, \quad j<m-\left[\frac{n}{2}\right] .
$$

which in turn yields,

$$
\sum_{|\beta|=j}\left|D^{\beta} U\right|_{\infty} \leq C(t+1)^{-\frac{n}{4}}, \quad \text { with } j<m-\left[\frac{n}{2}\right] .
$$

\section{PRELIMINARY ESTIMATES}

In this section we begin with some preliminary estimates for the heat system. If $V(x, t)$ is the solution to the homogeneous heat system, i.e., $V$ satisfies

$$
\left\{\begin{array}{l}
V_{t}(x, t)=\Delta V(x, t) \\
V(x, 0)=U_{0}(x)
\end{array}\right.
$$

Then we have the following

THEOREM 4.1. - Let $U_{0} \in L_{1} \cap L_{2}\left(\mathbb{R}^{n}\right) \cap R_{\alpha}^{\delta_{1}}$ for some $\alpha, \delta_{1}>0$, where

$$
R_{\alpha}^{\delta_{1}}=\left\{U:|\widehat{U(\xi)}| \geq \alpha, \text { for }|\xi| \leq \delta_{1}\right\}
$$

Then

$$
\int_{\mathbf{R}^{n}}|V(x, t)|^{2} d x \geq C(t+1)^{-\frac{n}{2}} \quad \text { and } \quad|\nabla V|_{\infty} \leq C t^{-\left(\frac{n}{1}+1\right)} .
$$

Proof. - See Schonbek [3]. 
THEOREM 4.2. - Let $V$ be a solution to the heat system with data $U_{0}$. Suppose that

$$
|V(t)|_{2}^{2} \leq C(t+1)^{-p}
$$

Then,

$$
\left|D^{s} V\right|_{\infty}<C(t+1)^{-\frac{n}{4}-\frac{\rho}{2}-\frac{s}{2}} \quad s \geq 0
$$

Proof. - Let $|V(t)|_{2}^{2} \leq C(t+1)^{-\rho}$ then it follows that

$$
\begin{aligned}
\left|D^{s} V\right|_{\infty} & \leq \int\left|\hat{D}^{s} V\right| d \xi \\
& \leq \int|\xi|^{2 s} \hat{V}(t / 2) e^{-|\xi|^{2} t / 2} d \xi \\
& \leq\left(\int|\xi|^{2 s} e^{-|\xi|^{2} t} d \xi\right)^{\frac{1}{2}}\left(\int_{\mathbf{R}^{n}}|\hat{V}(t / 2)|^{2} d \xi\right)^{\frac{1}{2}} \\
& \leq C_{1}\left((t+1)^{-\frac{n}{2}-s}\right)^{\frac{1}{2}}(t+1)^{-\frac{\rho}{2}} \\
& \leq C_{1}(t+1)^{-\frac{n}{4}-\frac{s}{2}-\frac{\rho}{2}}
\end{aligned}
$$

Proposition 4.3. - Let $V_{0} \in H^{s} \cap L_{1}$. This implies that if $V$ is a solution to the heat system with data $V_{0}$ then

$$
\left|D^{s} V\right|_{2}^{2} \leq C(t+1)^{-\frac{n}{2}-s}
$$

Proof. - The proof is standard and we inclue it for completeness. The $s$-derivatives of the solution to the heat equation can be explictly given by

$$
D^{s} V(x, t)=(4 \pi t)-\frac{n}{2} \int_{\mathrm{R}^{n}} D^{s} \exp \left(-\frac{|x-y|^{2}}{4 \pi t}\right)\left|V_{0}(y)\right|^{2} d y .
$$

Vol. $12, n^{\circ}$ 4-1995, 
Hence

$$
\begin{aligned}
\int_{\mathbf{R}^{n}}\left|D^{s} V(x, t)\right|^{2} d x \leq & (4 \pi t)^{-\frac{n}{2}} \int_{\mathbf{R}^{n}} \int_{\mathbb{R}^{n}}(4 \pi t)^{-\frac{n}{2}} D^{s} \\
& \times \exp \left(-\frac{|x-y|^{2}}{4 \pi t}\right)\left|V_{0}(y)\right|^{2} d y d x \\
\leq & (4 \pi t)^{-\left(\frac{n}{2}+s\right)} \int_{\mathbb{R}^{n}}(4 \pi t)^{-\frac{n}{2}} \\
& \times \exp \left(-\frac{|x-y|^{2}}{2 \pi t}\right) d x \int_{\mathbb{R}^{n}}\left|V_{0}(y)\right|^{2} d y \\
\leq & C(t+1)^{-\frac{n}{2}-s},
\end{aligned}
$$

which completes the proof of the proposition.

COROLlaRY 4.4. - If $|V|_{2}^{2} \leq C(t+1)^{-p}$, then

$$
\int_{\mathbf{R}^{n}}\left|\widehat{D^{s} V}\right|^{2} d \xi \leq C(t+1)^{-\frac{n}{2}-\rho-s}
$$

\section{UPPER BOUNDS FOR THE DIFFERENCE}

In this section we discuss bounds for the difference of the solution to the KdVB system and that of the heat system corresponding to the same initial data. There are several ways to approach this problem. Our approach, will be the Fourier splitting method ([3], [4]).

We first consider the case when the average of $U$ is non-zero. That is, we have $\int U_{0} d x \neq 0$. Let $R_{1}$ be a set defined by

$$
R_{1}=\left\{U_{0}: \int_{\mathbf{R}^{n}} U_{0} d x \neq 0\right\} .
$$

This implies that $U \in R_{\chi}^{\delta_{1}}$ where $R_{\chi}^{\delta_{1}}$ is defined by

$$
R_{\chi}^{\delta_{1}}=\left\{U:|\widehat{U(\xi)}| \geq \chi, \text { for }|\xi| \leq \delta_{1}\right\}
$$

We now obtain upper bounds for the solution to the KdVB system. But first, we need the following result. 
THEOREM 5.1. - Let the initial data $U_{0}$ be such that $U_{0} \in L_{1} \cap R_{1} \cap H^{p+1}$. Then there exists an upper bound for the difference between the solutions to the KdVB system and the heat system, both corresponding to the same initial data $U_{0}$. Thus if $p>1$ and if $W=U-V$ where $U$ is the solution to the KdVB system and $V$ that to the heat system, then $W$ satisfies,

$$
|W(t)|_{2}^{2} \leq C_{0}(t+1)^{-\left(\frac{n}{2}+1\right)}
$$

Proof. - We consider the difference $W=U-V$, where $U$ is the solution to the KdVB system, and $V$ that of the heat system, both corresponding to the same initial data $U_{0}$. Then $W$ satisfies the equation

$$
W_{t}-\Delta W=-\delta \delta_{2 p} U-\delta \operatorname{grad} \Phi(U)
$$

Multiply equation (32) by $W$ and integrate over space. Noting that $\int U \delta \delta_{2 p} U=0$ and $\int U \delta \operatorname{grad} \Phi(U)=0$, we obtain

$$
\begin{aligned}
& \frac{d}{d t} \int_{\mathbb{R}^{n}}|W|^{2} d x \\
& \leq-2 \int_{\mathbb{R}^{n}}|\delta W|^{2} d x \\
&+2|\delta V|_{\infty} \int_{\mathbf{R}^{n}}|\operatorname{grad} \Phi(U)| d x \\
&+2\left(\int_{\mathbb{R}^{n}}\left|\delta_{2 p+1} V\right|^{2} d x\right)^{\frac{1}{2}}\left(\int_{\mathbb{R}^{n}}|U|^{2} d x\right)^{\frac{1}{2}} .
\end{aligned}
$$

Using Plancherel's theorem the above inequality can be written in the form,

$$
\begin{aligned}
\frac{d}{d t} \int_{\mathbf{R}^{n}}|\hat{W}|^{2} d x \\
\leq-2 \int_{\mathbf{R}^{n}}|\xi|^{2}|\hat{W}|^{2} d \xi \\
\quad+2|\delta V|_{\infty} \int_{\mathbf{R}^{n}}|\operatorname{grad} \Phi(U)| d x \\
\quad+2\left(\int_{\mathbf{R}^{n}}\left|\delta_{2 p+1} V\right|^{2} d x\right)^{\frac{1}{2}}\left(\int_{R^{n}}|U|^{2} d x\right)^{\frac{1}{2}}
\end{aligned}
$$


Using the Fourier Splitting method, with

$$
S(t)=\left\{\xi:|\xi| \leq\left(\frac{\gamma}{2(t+1)}\right)^{\frac{1}{2}}\right\}
$$

with $\gamma$ sufficiently large, equation (34) can be rewritten as,

$$
\begin{aligned}
\frac{d}{d t} \int_{\mathbb{R}^{n}}|\hat{W}|^{2} d \xi= & -2 \int_{S(t)}|\xi|^{2}|\hat{W}|^{2} d \xi-2 \int_{S^{c}(t)}\left|\xi^{2}\right||\hat{W}|^{2} d \xi \\
& +2|\delta V|_{\infty} \int_{\mathbb{R}^{n}}|\operatorname{grad} \Phi(U)|^{2} d x \\
& +2\left(\int_{\mathrm{R}^{n}}\left|D^{2 p+1} V\right|^{2} d x\right)^{\frac{1}{2}}\left(\int_{\mathrm{R}^{n}}|U|^{2} d x\right)^{\frac{1}{2}}
\end{aligned}
$$

Hence, this can be simplified to yield,

$$
\begin{aligned}
\frac{d}{d t} \int_{\mathbb{R}^{n}}|\hat{W}|^{2} d \xi \leq & -\frac{\gamma}{(t+1)} \int_{S^{c}(t)}|\hat{W}|^{2} d \xi-2 \int_{S(t)}|\xi|^{2}|\hat{W}|^{2} d \xi \\
& +2|D V|_{\infty} \int_{\mathbb{R}^{n}}|\operatorname{grad} \Phi(U)| d x \\
& +2\left(\int_{\mathbb{R}^{n}}\left|D^{2 p+1} V\right|^{2} d x\right)^{\frac{1}{2}}\left(\int_{\mathbb{R}^{n}}|U|^{2} d x\right)^{\frac{1}{2}} .
\end{aligned}
$$

Further simplification yields,

$$
\begin{aligned}
& \frac{d}{d t}\left[(t+1)^{\gamma} \int_{\mathrm{R}^{n}}|W|^{2} d x\right] \\
& \leq n(t+1)^{\gamma-1} \int_{S(t)}|\hat{W}|^{2} d \xi \\
& \quad+C(t+1)^{\gamma}|D V|_{\infty} \int_{\mathbb{R}^{n}}|\operatorname{grad} \Phi(U)| d x \\
& \quad+C(t+1)^{\gamma}\left(\int_{\mathrm{R}^{n}}\left|D^{2 p+1} V\right|^{2} d x\right)^{\frac{1}{2}}\left(\int_{\mathbb{R}^{n}}|U|^{2} d x\right)^{\frac{1}{2}}
\end{aligned}
$$


Using the bounds obtained in (30) and (31) for the solution of the heat equation and the growth condition for $\Phi$ expressed in (5), we obtain,

$$
\text { (35) } \begin{aligned}
\frac{d}{d t} & {\left[(t+1)^{\gamma} \int_{\mathrm{R}^{n}}|W|^{2} d x\right] } \\
\leq & \gamma(t+1)^{\gamma-1} \int_{S(t)}|\hat{W}|^{2} d \xi \\
& +C(t+1)^{\gamma}(t+1)^{-\left(\frac{n}{4}+\frac{1}{2}\right)} C_{0} \cdot\left(|U|_{2}^{2}|U|_{\infty}^{\frac{4 p}{n}-1}\right) \\
& +C(t+1)^{\gamma}(t+1)^{-\left(\frac{n}{4}+p+\frac{1}{2}\right)}|U|_{2} \\
\leq & \gamma(t+1)^{\gamma-1} \int_{S(t)}|\hat{W}|^{2} d \xi+C_{1}(t+1)^{\gamma_{1}}+C_{2}(t+1)^{\gamma_{2}}
\end{aligned}
$$

supposing first that $p>1$ then $\gamma_{1} \geq \gamma_{2}=\gamma-\left(\frac{n}{2}+p+\frac{1}{2}\right)$. We now need to estimate $|\hat{W}|$ on the set $S(t)$. Taking the Fourier transform of (32) we have,

$$
\left\{\begin{array}{l}
\hat{W}_{t}+|\xi|^{2} \hat{W}=-\delta \widehat{\delta_{2 p} U}-\delta \operatorname{grad} \Phi(\mathrm{U})=-\hat{\mathrm{H}} \\
\hat{W}(\xi, 0)=0
\end{array}\right.
$$

Hence, it follows that

$$
\hat{W}(\xi, t)=-\int_{0}^{t} e^{-|\xi|^{2}(t-s)} \hat{H}(\xi, s) d s .
$$

If $\nu$ denotes the minimum power of $U_{i}$ in the definition of $\Phi$ then,

$$
|\hat{H}| \leq C|\xi|\left(|U|_{2}^{2}\left(|U|_{\infty}^{\nu-2}+|U|_{\infty}^{\frac{4 p}{n}-1}\right)+|\xi|^{2 p}|\hat{U}|_{\infty}\right)
$$

Since $|\hat{U}|$ is bounded by a constant depending only on the initial data and since $\xi \in S(t),|\xi| \leq\left(\frac{n}{(t+1)}\right)^{\frac{1}{2}}$. Moreover, with $p \geq 1$ and $n \geq 2$ it follows that

$$
\begin{aligned}
|W \widehat{(\xi, t)}|_{\infty} & \leq \frac{C}{(t+1)^{\frac{1}{2}}} \int_{0}^{t}\left[(s+1)^{-\left(p+\frac{n}{4}\right)}+(s+1)^{-\frac{\nu n}{4}}+C(s+1)^{-p}\right] d s \\
& \leq \frac{C}{(t+1)^{\frac{1}{2}}} .
\end{aligned}
$$

Vol. $12, \mathbf{n}^{\circ} 4-1995$. 
Thus,

$$
|W \widehat{(\xi, t)}|_{\infty} \leq \frac{C}{(t+1)^{\frac{1}{2}}}
$$

Substituting (38) into (35) and simplifying the result we obtain

$$
\frac{d}{d t}\left[(t+1)^{\gamma} \int_{\mathbf{R}^{n}}|W|^{2} d x\right] \leq C(t+1)^{\gamma-1}(t+1)^{-1-\frac{n}{2}}+C_{1}(t+1)^{\gamma_{2}}
$$

since $\gamma_{1}>\gamma_{2}$. Integrating over the interval $[0, t]$ yields,

$$
(t+1)^{\gamma} \int_{\mathbf{R}^{n}}|W(t)|^{2} d x \leq C_{1}(t+1)^{\gamma-\left(\frac{n}{2}+1\right)}
$$

or equivalently,

$$
\int_{\mathbb{R}^{n}}|W(t)|^{2} d x \leq(t+1)^{-\left(\frac{n}{2}+1\right)}
$$

This proves the theorem.

\section{LOWER BOUNDS}

In obtaining the lower bounds for the solution to the KdVB system, two cases have to be considered. In the first case the mean of the initial data is different from zero, that is long waves are present. In this case the lower bound for the decay rate for the solution to the heat system is $(t+1)^{-\frac{n}{2}}$. Hence from the previous section we note that as a consequence of the upper bounds for the difference of solutions to the heat equation and the KdVB system, which is of order $(t+1)^{-\left(\frac{n}{2}+1\right)}$ when $p>1$, the lower bounds for the solution to the KdVB system follow easily. That is since

$$
|U(t)|_{2}^{2} \geq|V|_{2}^{2}-|W|_{2}^{2}
$$

it follows that,

$$
|U(t)|_{2}^{2} \geq C(t+1)^{-\frac{n}{2}}
$$


The second case to be considered is when the mean of the initial data equals zero. In Fourier space this is equivalent to the statement that the Fourier transform of the initial data has a zero at the origin. The bounds that are obtained in this case for the solution to the heat system depend on the order of the zero. We will show that outside of a class of data to be defined below the solution to the KdVB system will have a uniform rate of decay of order $\frac{n}{2}+1$, showing that in the far-field the behaviour of the solutions to the Heat system and that to the KdVB system are very different, i.e., long waves can be created for such systems, a phenomenon which is absent in the Heat system. Before we begin estimates for the lower bound, some preliminary results are needed. We begin by stating the following result, proved by Schonbek [4].

TheOREM 6.1. - Let $V_{0} \in L_{2}\left(\mathbb{R}^{n}\right)$. Let $V$ be a solution to the heat system with initial data $V_{0}$. Suppose that there exists functions $l$ and $h$ such that the Fourier transform of $V_{0}$ admits, for $|\xi| \leq \delta_{1}$ with $\delta_{1}>0$ the representation

$$
\hat{V}_{0}(\xi)=\xi \cdot l(\xi)+h(\xi), \quad l=\left(l_{1}, \ldots, l_{n}\right),
$$

where $l$ and $h$ satisfy the following conditions:

(i) $|h(\xi)| \leq M_{0}|\xi|^{2}$, for some $M_{0}>0$.

(ii) $l$ is homogeneous of degree zero.

(iii) $\gamma=\int_{|\omega|=1}|\omega \cdot l(\omega)|^{2} d \omega>0$.

Let $M_{1}=\sup _{|y|=1}|l(y)|, M_{2}=\sup _{\frac{\delta_{1}}{2} \leq|y| \leq 1}|\nabla l(y)|, K=\max \left(M_{0}\right.$, $\left.M_{1}, M_{2}\right)$. Then there exist constants $C_{0}$ and $C_{1}$ such that

$$
C_{0}(t+1)^{-\left(\frac{n}{2}+1\right)} \leq|V(\cdot, t)|_{2}^{2} \leq C_{1}(t+1)^{-\left(\frac{n}{2}+1\right)}
$$

where $C_{0}$ and $C_{1}$ both depend on $n, M_{0}, M_{1}, \delta_{1}$ and $\left|V_{0}\right|_{2}$ and $C_{0}$ also depends on $K$ and $\alpha_{1}$. Note that condition (iii) is not necessary for the upper bound of $|V(\cdot, t)|_{2}$.

Proof. - See Schonbek [4].

Vol. $12, \mathrm{n}^{\circ} 4-1995$. 
Let

$$
\begin{aligned}
\mathcal{M}_{\mathcal{R}} & =\{U: \operatorname{Re} \widehat{\nabla U}(0)=0\} \\
\mathcal{M}_{\mathcal{I}} & =\{U: \operatorname{Im} \widehat{\nabla U}(0)=0\} \\
P_{k} & =\int_{0}^{\infty} \widehat{\Phi_{U_{K}}}(U(0, s)) d s=\int_{0}^{\infty} a_{k}^{0} d s \\
\bar{P}_{k} & =\left(P_{k}, \ldots, P_{k}\right) \text { and } \\
\hat{\Phi}(U) & =\left[\int \Phi e^{-2 \pi i x \xi} d x\right]
\end{aligned}
$$

It the Fourier transform of the initial data has a zero of order one and $U_{0} \in W_{1}$ then, this implies that either $U_{0} \notin \mathcal{M}_{\mathcal{R}}$ and $U_{0} \notin \mathcal{M}_{I}$.

THEOREM 6.2. - Let $U_{0} \in W_{1} \cap L_{1} \cap H^{p+1}$. Suppose that $\int U_{0} d x=0$ and $\hat{U}_{0}$ has a zero of order one. Let $p>1$ and let $U$ be a solution to the $K d V B$ system with data $U_{0}$. Suppose that

(i) $U_{0} \notin \mathcal{M}_{R}$ or

(ii) For some $k$,

$$
\operatorname{Im} \widehat{\nabla U}_{k}(0)-P_{k} \neq 0
$$

Then there exist constants $C_{1}$ and $C_{2}$ such that

$$
C_{1}(1+t)^{-\left(\frac{n}{2}+1\right)} \leq|V(\cdot, t)|_{2}^{2} \leq C_{2}(1+t)^{-\left(\frac{n}{2}+1\right)}
$$

for all $t \geq 0$ where $V$ is the solution to the heat equation with data $U_{0}$ and there exist constants $K_{1}$ and $K_{2}$ such that

$$
K_{1}(1+t)^{-\left(\frac{\pi}{2}+1\right)} \leq|U(\cdot, t)|_{2}^{2} \leq K_{2}(1+t)^{-\left(\frac{n}{2}+1\right)}
$$

where $C_{1}, C_{2}, K_{1}, K_{2}$ depend only on the norms of the data.

Proof. - We first need to explain our hypotheses. They ensure that long waves persits, i.e., we associate long waves with the terms of order one in the Fourier expansion. Note that

$$
\widehat{U(\xi, t)}=\hat{U}_{0}+\xi \widehat{\nabla U}_{0}+i \xi P+\mathcal{O}\left(|\xi|^{2}\right) .
$$

If we choose data which satisfies $A$ or $B$ below, then the first order terms in the Taylor expansion of the Fourier transform persits, i.e., long waves 
will persist. Since the hypotheses are such that $\hat{U}_{0}(\xi)$ has a zero of order one, we need that

$$
P_{k}-\operatorname{Im} \widehat{\nabla U}_{0} \neq 0 \text {. or } \operatorname{Re} \widehat{\nabla U}_{0} \neq 0 .
$$

Note that the following conditions on the data and on the function $\Phi$ ensure that (40) holds.

A) $U_{0} \notin \mathcal{M}_{R}^{c}$.

B) $U_{0} \in \mathcal{M}_{I}$ and $\Phi(U)=\sum_{i} \sum_{k} m_{k_{i}} U_{i}^{k_{i}}$ with $m_{k_{i}}=0$ for $k$ odd.

We begin by considering the KsVB system. The nonlinear term $\delta \operatorname{grad} \Phi(U)$ is such that

$$
\widehat{\operatorname{grad} \Phi}(U)=\left(\widehat{\Phi_{U_{1}}}, \widehat{\Phi_{U_{2}}}, \ldots, \widehat{\Phi_{U_{n}}}\right)
$$

We introduce the notation

$$
a_{i}=\widehat{\Phi_{U_{i}}}
$$

Hence we have

$$
\widehat{\operatorname{grad} \Phi}(U)=\left(a_{1}, \ldots, a_{n}\right)
$$

Taking the Fourier transform of the KdVB system we arrive at,

$$
\begin{gathered}
\hat{U}_{t}+|\xi|^{2} \hat{U}=-\hat{H}=-\delta \operatorname{grad} \Phi(U)-\delta \widehat{\delta_{2 p} U} \\
\hat{U}(\xi, 0)=\hat{g}(\xi) .
\end{gathered}
$$

Treating this as an ordinary differential equation for $U$ and writing down the formal solution we obtain for each component, the equation

$$
\hat{U}_{k}(\xi, t)=g_{k}(\xi) e^{-|\xi|^{2} t}-\int_{0}^{t} \hat{H}_{k}(\xi, s) e^{-|\xi|^{2}(t-s)} d s
$$

where,

$$
\hat{H}_{k}(\xi, s)=-i \sum_{j=1}^{n} \xi_{j}(\operatorname{grad} \Phi(U))_{k}-i \sum_{j=1}^{n} \xi_{j}|\xi|^{2 p} \hat{U}_{k}
$$

This enables us to rewrite it in the form,

$$
\hat{H}_{k}(\xi, s)=-i \sum_{s} \xi_{s} \cdot\left[a_{k}+\sum_{j} \xi_{j}^{2 p} \hat{U}_{k}\right]
$$


which implies that

$$
\hat{H}=\left(\hat{H}_{1}, \ldots, \hat{H}_{n}\right)=i \xi \cdot\left(\tilde{a}_{k}-\sum_{j} \xi_{j}^{2 p} \tilde{\hat{U}}_{k}\right)
$$

where

$$
\tilde{a}_{k}=\left(a_{k}, \ldots, a_{k}\right)
$$

and

$$
\tilde{\hat{U}}_{k}=\left(\hat{U}_{k}, \ldots, \hat{U}_{k}\right)
$$

By the hypothesis on the form of $\delta \operatorname{grad} \Phi$ it follows that,

$$
\hat{H}_{k}(\xi, s)=-i \sum_{j=1}^{n} \xi \cdot\left[a_{k}+|\xi|^{2 p} \hat{U}_{k}\right]
$$

Let $a_{i}^{0}=a_{i}(0, t)$. Then $a_{i}$ can be represented as

$$
a_{i}=a_{i}^{0}+\xi \cdot \nabla_{\xi} a_{i}(\bar{\xi}), \quad 0 \leq \bar{\xi} \leq \xi
$$

In the appendix (Theorem Al) we show that for $|\xi| \leq \delta_{1}$

$$
\left|\nabla_{\xi} a_{i}(\xi, t)\right| \leq C(t)
$$

where $C(t)$ denotes a constant independent of $\xi$ but which depends on $|g|_{2},|g|_{W_{2}}, \delta_{1}$ and $t$. Therefore it is possible to write $\hat{H}_{k}$ as

$$
\hat{H}_{k}(\xi, s)=-i \sum_{j=1}^{n} \xi_{j} \cdot\left[a_{k}^{0}+\xi \cdot \nabla_{\xi} a_{k}(\bar{\xi})+|\xi|^{2 p} \hat{U}_{k}\right] .
$$

This yields

$$
\int_{0}^{t} \hat{H}_{k}(\xi, s) e^{-|\xi|^{2}(t-s)} d s=i \sum_{j} \xi_{j} \cdot \int_{0}^{t} a_{k}^{0} d s+\text { H.O.T. }
$$

where

$$
\text { H.OT. }=\int_{0}^{t} a_{k}^{0}\left(e^{-|\xi|^{2}(t-s)}-1\right) d s+i \xi \cdot \int_{0}^{t}\left[\nabla_{\xi} a_{k}+|\xi|^{2 p} \hat{U}_{k}\right] d s .
$$


Hence it follows that

$$
\hat{U}_{k}(\xi, t)=-i \xi \cdot L_{k}\left(\xi, t_{0}\right)+(\text { H.O.T. })_{k}
$$

with

$$
L_{k}\left(\xi, t_{0}\right)=\left(\int_{0}^{t} a_{k}^{0} d s, \ldots, \int_{0}^{t} a_{k}^{0} d s\right)
$$

and

$$
(\text { H.O.T. })_{k}=\hat{g_{k}}(\xi) e^{-|\xi|^{2} t}+a_{k}^{0}\left(e^{-|\xi|^{2}(t-s)}-1\right)+i \xi \cdot\left[\nabla_{\xi} a_{k}+|\xi|^{2 p} \hat{U}_{k}\right] \text {, }
$$

where

$$
\int_{0}^{t} a_{k}^{0}=\int_{0}^{t} \Phi_{U_{k}} d s=\int_{0}^{t} U_{k}^{j_{k}-1} d s .
$$

To apply Theorem 6.1 we need a lower bound for $\alpha_{k}$, for at least one $k$, where

$$
\alpha_{k}(t)=\alpha_{k}=\int_{|\omega|=1}\left|\omega \cdot L_{k}(\omega(t))\right|^{2} d \sigma
$$

and

$$
L_{k}(\omega, t)=\left[\int_{0}^{t} a_{k}^{0}, \ldots, \int_{0}^{t} a_{k}^{0}\right] .
$$

Conditions (i), (ii) ensure that there exists a sequence $t_{n} \rightarrow \infty$ such that (making use of the notation introduced above), for some $\xi_{0}$

$$
U_{k}\left(\xi_{0}, t_{n}\right)=\xi_{0} \cdot L_{k}\left(t_{n}\right)+\text { H.O.T. }
$$

where $L_{k}\left(t_{n}\right)=\operatorname{Re} \widehat{\nabla U}(0)+i\left(P_{k}-\operatorname{Im} \widehat{\nabla U}(0)\right)$ and for some $\xi_{0}$

$$
\xi_{0} \cdot L_{k}\left(t_{n}\right) \geq \alpha>0,
$$

with $\alpha$ given by $\alpha=\max \left\{|\operatorname{Re} \widehat{\nabla U}(0)|,\left|P_{k}-\operatorname{Im} \widehat{\nabla U}(0)\right|\right\}$, that is $\alpha$ is independent of $t_{n}$. Moreover, $\xi_{0}$ can be chosen to be of the form

$$
\xi_{0}=(0, \ldots, 1, \ldots, 0)=e_{j}
$$

for $j=k$ so that (40) holds.

Vol. $12, \mathrm{n}^{\circ} 4-1995$. 
A) and B) above show that the class of data producing solutions which satisfy (42) and (43) is large. Moreover, note that there exists $N\left(\xi_{0}\right)$ independent of $t_{n}$ such that $\xi \cdot L_{k}\left(t_{n}\right) \geq \frac{\alpha}{2}$ for $\xi \in N\left(\xi_{0}\right) \cap S^{n-1}$. This can be shown as follows. First we show that for $k-1>2,\left|L_{k}(t)\right| \leq C$, which follows since,

$$
\int_{0}^{t} \int\left|a_{k}^{0}\right| \leq C \int_{0}^{t} \int|U|^{k_{i}-1} \leq C \int_{0}^{t} \frac{1}{(s+1)^{\frac{n}{2}+1}} \leq C .
$$

Let

$$
\xi=(\varepsilon, \ldots, \varepsilon, 1-\sqrt{n-1} \varepsilon, \ldots, \varepsilon) .
$$

and it follows that $|\xi|=1$ and $\left|\xi-\xi_{0}\right| \leq \tilde{\varepsilon}=2 \sqrt{n-1} \varepsilon$. This implies that

$$
\xi \cdot L_{k}\left(t_{n}\right)>\frac{\alpha}{2} .
$$

Note that if $\operatorname{Re} \widehat{\nabla U}_{0} \neq 0$ then it follows that

$$
\left|\xi \cdot L_{k}\right|^{2}=\left|\operatorname{Re} \widehat{\nabla U}_{0}\right|^{2}+\left|\operatorname{Im} \widehat{\nabla U}_{0}-\int_{0}^{t} \bar{P}_{k}\right|^{2} \geq\left|\operatorname{Re} \widehat{\nabla U}_{0}\right|^{2} \geq \alpha / 2
$$

Also note that,

$$
\alpha\left(t_{n}\right)=\alpha_{1}=\int_{|\omega|=1}\left|\xi \cdot L_{k}\left(t_{n}\right)\right|^{2} \geq \frac{\alpha^{2}}{4} \omega_{n}=\rho
$$

where $\omega_{n}$ denotes the volume of the unit sphere. By the above remarks

$$
\hat{U}(\xi, T)=\xi \cdot L(\xi, T)+H(\xi, T),
$$

for $T=t_{n}$ and $|\xi L(\xi, T)| \geq C_{0}$ for all $n$. Recall that for $t \geq \delta_{0}$, (see Theorem 6.1) $\delta_{0}=\left(\delta_{0}\left(\alpha_{1}\left(t_{n}\right)\right)\right)$ where $\delta$ is defined by the requirement that $4 \delta M_{0} M_{1} \leq \alpha_{1}$, since we can clearly choose $\delta_{1} \leq \delta_{0}\left(t_{n}\right)$ for all $n$ by letting $4 \delta_{1} M_{0} M_{1}=\rho$. (see Schonbek [4].) Then $V(x, t)$ the solution to the heat system with data $U_{0}$ satisfies

$$
|V(\cdot, t)|_{2}^{2} \geq \chi_{\rho}(t+1)^{-\left(\frac{n}{2}+1\right)}
$$

for $t \geq t_{k}$, with $\chi_{\rho}=\frac{\alpha \omega_{n} e^{-1}}{2(n+2)}$ (cf. Schonbek [4]), which, by the computations above is independent of $t_{k}$. 
Let $v$ be the solution to the heat system with initial data $v(x, 0)=$ $U\left(x, T_{1}\right)$ such that $K\left(1+T_{1}\right)^{-\theta} \leq \frac{\chi_{\rho}}{8} \omega_{0}$, where $K=K_{1} K_{2}^{\frac{4 p}{n}-1} K_{3}$ and $\chi_{\rho}$ is as above. The constants $K_{1}, K_{2}$ and $K_{3}$ are defined as below.

$$
\begin{aligned}
|\operatorname{grad} \Phi| & \leq K_{1}|U|_{2}^{2}\left(|U|^{\frac{4 p+1}{\infty^{n}}}+|U|_{\infty}^{\nu}\right) \\
|U|_{2}^{2} & \leq K_{3}(1+t)^{-\frac{n}{2}} \\
|U|_{\infty} & \leq K_{2}(1+t)^{-\frac{n}{4}}
\end{aligned}
$$

and $\omega_{0}$ denotes the surface area of $S^{n-1}$, and

$$
\theta=\min \left\{\frac{n}{4}+\frac{n}{2}-1, p+\frac{n}{4}-1\right\} .
$$

Thus by Theorem 6.1 it follows that for $t \geq \delta_{1}=\delta_{1}(\rho)$

$$
\chi_{\rho}(t+1)^{-\left(\frac{n}{2}+1\right)} \leq|v(\cdot, t)|_{2}^{2} \leq K_{0}(t+1)^{-\left(\frac{n}{2}+1\right)}
$$

where $K_{0}$ depends on the $L_{2}$ norm of $U_{0}$ and $\chi_{\rho}$ depens on $\beta$ and $C_{0} . \delta_{0}$ is independent of $T_{1}$ since $\delta$ dpends on $\rho$ by the uniformity condition (44).

Let

$$
u(x, t)=U\left(x, t+T_{1}\right) .
$$

The difference $w=v-u$ is now studied. The decay rates for the KdVB system will imply that $|w(\cdot, t)| \leq C(t+1)^{-\left(\frac{n}{2}+1\right)}$ with $C$ sufficiently small. $W$ satisfies an inhomogeneous heat system. The Fourier splitting method will yield

$$
\begin{aligned}
& \frac{d}{d t}\left[(t+1)^{8 n} \int_{\mathbb{R}^{n}}|w|^{2} d x\right] \\
& \leq(t+1)^{8 n-1} \int_{S(t)}|\hat{w}|^{2} d \xi+K_{*}\left|D^{2 p+1} v\right|_{2}|u|_{2} \\
& \quad+K_{2}|\nabla v|_{\infty} \int_{\mathbb{R}^{n}}|u|^{\frac{4 p}{n}+1} d x .
\end{aligned}
$$

The second and third terms on the right-hand side of the above inequality will be of higher order and will as such be easily bounded as follows:

$$
\begin{array}{ll}
K_{2}|\nabla v|_{\infty} \int_{\mathrm{R}^{n}}|u|^{\frac{4 p}{n}+1} d x & \leq M_{1}(t+1)^{-\gamma_{1}} \\
K_{*}\left|D^{2 p+1} v\right|_{2}|u|_{2} & \leq M_{2}(t+1)^{-\gamma_{2}}
\end{array}
$$


where $\gamma_{1}=\frac{n}{2}+p+\frac{1}{2}=\gamma_{2}$. In obtaining these bounds, we make use of the bounds on the derivatives of the solution to the heat equation and the $L_{\infty}$ and $L_{2}$ bounds for the solution to the KdVB system. To bound the first term on the right-hand side of (46) the Fourier transform of the inhomogeneous heat equation yields

$$
\hat{w}+|\xi|^{2} \hat{\omega}=-\delta \operatorname{grad} \Phi(u)-\delta \widehat{\delta_{2 p} u}=-\hat{H}
$$

As before, in (37)

$$
\begin{aligned}
|\hat{H}(\xi, t)| \leq & K_{1}|\xi|\left(|u(\cdot, t)|_{\infty}^{\frac{4 p}{n}-1}+|u(\cdot, t)|_{\infty}^{\nu-2}\right)|u(\cdot, t)|_{2}^{2} \\
& +K_{*}|\xi|^{2 p+1}|\hat{u}(\cdot, t)|_{\infty} .
\end{aligned}
$$

Let $\nu=\min$ power of $U_{i}$ in $\Phi(U)$. Therefore,

$$
\begin{aligned}
|\hat{w}(\xi, t)| \leq & \int_{0}^{t}|\hat{H}(\xi, s)| d s \\
\leq & K_{1}|\xi| \int_{0}^{t}\left(|u(\cdot, s)|_{\infty}^{\nu-2}+|u(\cdot, s)|_{\infty}^{\frac{4 p}{n}-1}\right)|u(\cdot,)|_{2}^{2} d s \\
& \quad+K_{*}|\xi|^{2 p+1} \int_{0}^{t}|\hat{u}(\cdot, s)|_{\infty} d s \\
= & K_{1}|\xi| \int_{T_{1}}^{t+T_{1}}\left(|U(\cdot, s)|_{\infty}^{\nu-2}+|U|_{\infty}^{\frac{4 p}{n}-1}\right)|U(\cdot, s)|_{2}^{2} d s \\
& +K_{2}|\xi|^{2 p+1} \int_{T_{1}}^{t+T_{1}}|\hat{U}(\cdot, s)| \infty d s \\
\leq & K_{1}|\xi| \int_{T_{1}}^{t+T_{1}}\left(\frac{K_{2}^{(\nu-2)}}{\left.(s+1)^{\frac{n}{4} \nu}+\frac{K_{1}^{\frac{4 p}{n}}-1}{(1+s)^{p+\frac{n}{4}}}\right) d s}\right. \\
& \quad+C K_{*}|\xi|^{2 p+1} \int_{T_{1}}^{t+T_{1}} C^{*} d s \\
\leq & K_{1}|\xi|\left(\frac{1}{\left(1+T_{1}\right)}\right)^{\omega_{0}+C K_{*}|\xi|^{2 p+1} t}
\end{aligned}
$$


where $\theta=\min \left\{\frac{n}{4} \nu-1, p+\frac{n}{4}-1\right\}$. Since $n \geq 2$ and $p \geq 1$ we have $\theta>0$. Here we have made use of the fact that the $L_{\infty}$-norm of the solution to the KdVB system decays at the rate of $(1+t)^{-\frac{n}{4}}$.

Thus we have,

$$
\left.\left|\hat{w}(\xi, t) \leq C_{1}\right| \xi\left|+C_{2}\right| \xi\right|^{2 p+1} t
$$

where $C_{1}=C_{1}\left(T_{1}\right)=\frac{C K}{\left(1+T_{1}\right)^{\theta}}$ and $C_{2}=C K_{*}$. Note that $T_{1}$ can be chosen as large as needed since $\chi_{\rho}$ is independent of $T_{1}$. Using the definition of the set $S(t)$, this implies that

$$
\begin{aligned}
\int_{S(t)}|\hat{w}|^{2} d \xi & \leq C_{1} \frac{\omega_{0}}{n} \int_{S(t)}|\xi|^{2} d \xi+C_{2} t^{2} \int_{S(t)}|\xi|^{4 p+2} d \xi \\
& \leq C_{1}(t+1)^{-\left(\frac{n}{2}+1\right)}+C_{2}(t+1)^{-\left(\frac{n}{2}+2 p-1\right)}
\end{aligned}
$$

Hence the first term on the right-hand side of (46) can be bounded as follows.

$$
\begin{aligned}
(t+1)^{8 n-1} \int_{S(t)}|\hat{w}|^{2} d \xi & \leq C_{1}(t+1)^{8 n-\frac{n}{2}-2}+C_{2}(t+1)^{8 n-\frac{n}{2}-2 p} \\
& \leq \frac{\chi \rho}{8}(t+1)^{\frac{15 n}{2}-2}+C_{2}(t+1)^{\frac{15 n}{2}-2 p}
\end{aligned}
$$

The last inequality follows from the choice of $T_{1}$. Combining this last estimate with (46) and (47) yields

$$
\begin{aligned}
\frac{d}{d t}\left[(t+1)^{8 n} \int_{\mathbf{R}^{n}}|w|^{2} d x\right] \leq & \frac{\chi_{\rho}}{8}(t+1)^{\frac{15 n}{2}-2} \\
& +C_{2}(t+1)^{\frac{15 n}{2}-2}+M_{1}(t+1)^{(8 n-1)-\gamma_{1}} \\
& +M_{2}(t+1)^{(8 n-1)-\gamma_{2}} .
\end{aligned}
$$

Integrating over the interval $\left[\delta_{1}(\rho), t\right]$ gives

$$
\begin{aligned}
\int_{\mathbf{R}^{n}}|w|^{2} d x \leq & \frac{\chi_{\rho}}{8}(t+1)^{-\left(\frac{n}{2}+1\right)} \\
& +C_{2}(t+1)^{-\left(\frac{n}{2}+2 p-1\right)}+M_{1}(t+1)^{-\left(\frac{n}{2}+p+\frac{1}{2}\right)} \\
& +M_{2}(t+1)^{-\left(\frac{n}{2}+p+\frac{1}{2}\right)}+(t+1)^{-8 n} \int_{\mathbf{R}^{n}}\left|w\left(x, \delta_{1}\right)\right|^{2} d x
\end{aligned}
$$

Vol. $12, \mathrm{n}^{\circ}$ 4-1995. 
Note that

$$
\int_{\mathbf{R}^{n}}\left|w\left(x, \delta_{1}\right)\right|^{2} d x \leq \int_{\mathbf{R}^{n}}|u(x, 0)|^{2} d x+\int_{\mathbf{R}^{n}}|v(x, 0)|^{2} d x \leq 2 \int_{\mathbf{R}^{n}}\left|U_{0}\right|^{2} d x .
$$

Hence, it follows that

$$
\int_{\mathbb{R}^{n}}|w|^{2} \leq \frac{\chi_{\rho}}{8}(t+1)^{-\mu}+\text { H.O.T. }
$$

for $t$ large enough, where $\mu$ is defined by

$$
\mu=\frac{n}{2}+1 \text {. }
$$

The rest of the terms are of higher order since $2 p>n, \frac{n}{2}+1<\frac{n}{2}+p+\frac{1}{2}$ and $\frac{n}{2}+1<\frac{n}{2}+2 p-1$. That is for $t \geq T_{1}$

$$
\begin{aligned}
|u(\cdot, t)|_{2} & \geq|v(\cdot, t)|_{2}-|w(\cdot, t)|_{2} \\
& \geq \chi_{\rho}(t+1)^{-\left(\frac{n}{2}+1\right)}-\frac{\chi_{\rho}}{8}(t+1)^{-\left(\frac{n}{2}+1\right)}+\text { H.O.T }
\end{aligned}
$$

and $T_{2}$ is such that

$$
\text { H.O.T. }<\frac{\chi_{\rho}}{8}(t+1)^{-\frac{n}{2}-1} .
$$

Hence, for $t \geq T_{3}=T_{1}+T_{2}$

$$
|u(\cdot, t)|_{2} \geq \frac{\chi_{\rho}}{4}(t+1)^{-\frac{1}{2}\left(\frac{n}{2}+1\right)}
$$

For $t<T_{3}$ the decay of energy of $U$ yields

$$
|u(\cdot, t)|_{2}^{2} \geq\left|u\left(\cdot, T_{3}\right)\right|_{2}^{2} \geq \chi_{\rho} / 4\left[\frac{1+t}{1+T_{3}}\right]^{\frac{n}{2}+1}(1+t)^{-\left(\frac{n}{2}+1\right)}
$$

and the result follows. This completes the proof.

THEOREM 6.3. - Let $U_{0} \in L_{1} \cap H^{p+1} \cap \mathcal{M}_{I} \cap \mathcal{M}_{\mathcal{R}}$. Let $U$ be a solution of the KdVB system with data $U_{0}$ where $U_{0}$ is such that $\int U_{0}=0$ and $\hat{U}_{0}$ has a zero of order $>1$. If $\left|P_{k}\right| \neq 0$ for some $k$ then, there exists constants $K_{1}, K_{2}$ such that

$$
K_{1}(t+1)^{-\left(\frac{\pi}{2}+1\right)} \leq|U(\cdot, t)|_{2}^{2} \leq K_{2}(t+1)^{-\left(\frac{n}{2}+1\right)}
$$


where $K_{1}, K_{2}$ depend only on the norms of the data.

Proof. - The proof is similar to the case when the data is of order one. Note that if $U_{0} \in \mathcal{M}_{\mathcal{I}} \cap \mathcal{M}_{\mathcal{R}}$ we only need

$$
\begin{array}{ll}
\Phi(U)=\sum \alpha_{k_{i}} U_{i}^{k_{i}} & k_{i} \text { odd or } \\
\Phi(U)=\sum \alpha_{k_{i}} U_{i}^{k_{i}} & k_{i} \text { even, }
\end{array}
$$

Then, $P_{k} \neq 0$. As in the proof of Theorem 6.6, there exists a sequence of $t_{n}$ such that

$$
\hat{U}(\xi, t)=\xi \cdot L\left(t_{n}\right)+H . O . T .
$$

This implies the uniform condition for the lower bounds,

$$
\xi \cdot L_{k}\left(t_{n}\right) \geq \alpha / 2 \quad \text { for } \xi \in N\left(\xi_{0}\right) \cap S^{k-1}
$$

for some $\xi_{0}$, and the proof now is a repetition of the proof of Theorem 6.2.

\section{APPENDIX}

THEOREM A1. - Let $U_{0} \in H^{p+1} \cap W_{2}$. Then if $U$ is the solution to the $K d V B$ system with data $U_{0}$ then,

$$
\nabla_{\xi} a_{i}(\bar{\xi}) \leq C(t)
$$

where $C(t)$ depends only on the norm of $U_{0}$ in the spaces $H^{p+1}$ and $W_{2}$.

Proof. - By definition,

$$
a_{i}=\hat{\Phi}_{U_{i}}
$$

and

$$
\Phi_{U_{i}}=\left(\sum_{i} \sum_{k} m_{k_{i}} U_{i}^{k_{i}}\right)_{U_{i}}=\sum m_{k_{i}} U_{i}^{k_{i}-1} .
$$

We carry out the analysis of one term of the sum. Note that $k>2$ since otherwise the equation is linear. Thus, for $k>2$

$$
\left|\nabla_{\xi_{k}} a_{i}\right| \leq C \int\left|U_{i}^{k_{j}-1}\right|\left|x_{k}\right| \leq C \int U_{i}^{2}\left|x_{k}\right|
$$


When $k_{j}>2$ it suffices to bound $\int U_{i}^{2}|x| d x$. We have,

$$
\begin{aligned}
\frac{d}{d t} \int U_{i}^{2}\left|x_{k}\right|= & \int U_{i}\left|x_{k}\right| U_{i t} \\
= & \int\left|x_{k}\right| U_{i}(\delta \operatorname{grad} \Phi(U))_{i} \\
& +\int\left|x_{k}\right| U_{i} \delta_{2 p+1} U_{i}-\int\left|x_{k}\right| U_{i}(\Delta U)_{i} \\
= & I+I I+I I I .
\end{aligned}
$$

Note that

$$
\begin{aligned}
I=\int\left|x_{k}\right| U_{i}(\delta \operatorname{grad} \Phi)_{i}= & \int \operatorname{sgn}\left(x_{k}\right) U_{i} U_{i}^{k_{i}-1} \\
& +\sum_{j} \int\left|x_{k}\right| U_{i}^{k_{i}-1} U_{i} x_{j} \\
\leq & C_{0} \int U_{i}^{2}+\frac{1}{k} \int \operatorname{sgn}\left(x_{k}\right) U_{i}^{k_{j}} \\
\leq & C_{0}+\frac{C_{0}}{k} \int\left|U_{i}\right|^{2}
\end{aligned}
$$

and

$$
\begin{aligned}
I I=\int\left|x_{k}\right| U_{i} \delta_{2 p+1} U_{i}= & -\int \operatorname{sgn}\left(x_{k}\right) U_{i} \delta_{2 p} U_{i} \\
& -\int\left|x_{k}\right| \delta U_{i} \delta_{2 p} U_{i} \\
= & -\int \operatorname{sgn}\left(x_{k}\right) U_{i} \delta_{2 p} U_{i}+\int \operatorname{sgn}\left(x_{k}\right) \delta_{2 p-1} U_{i} \\
& +\int\left|x_{k}\right| \delta_{2} U_{i} \delta_{2 p-1} U_{i}
\end{aligned}
$$

Hence it follows that

$$
\begin{aligned}
I I= & \int \operatorname{sgn}\left(x_{k}\right) \sum_{k<2 p+1} \alpha_{k} \delta_{2 p+1-k} U_{i} \delta_{k} U_{i} \\
& +(-1)^{2 p+1} \int\left|x_{k}\right| \delta_{2 p+1} U_{i} U_{i}
\end{aligned}
$$


and we can conclude that

$$
\begin{aligned}
2 I I= & \int \operatorname{sgn}\left(x_{k}\right) \sum_{k<2 p+1} \alpha_{k} \delta_{2 p+1-k} U_{i} \delta_{k} U_{i} \\
& \leq \alpha_{k}\left(\int\left|D^{s} U_{i}\right|^{2}\right)^{\frac{1}{2}}\left(\int\left|D^{j} U_{i}\right|^{2}\right)^{\frac{1}{2}} \leq C
\end{aligned}
$$

where $s, j \leq 2 p+1$. Finally,

$$
\begin{aligned}
I I I=\int\left|x_{k}\right| U_{i} \Delta U_{i}= & -\sum_{j} \int\left|x_{k}\right| U_{i x_{j}}^{2}+\int \operatorname{sgn}\left(x_{k}\right) U_{i} \nabla U \\
& \leq\left(\int U_{i}^{2}\right)^{\frac{1}{2}}\left(\int\left|\nabla U_{i}\right|^{2}\right)^{\frac{1}{2}} \\
& \leq C
\end{aligned}
$$

Combining these estimates in (48) we obtain

$$
\frac{d}{d t} \int U_{i}^{2}\left|x_{k}\right| \leq C .
$$

Integrating this with respect to time the desired result follows.

\section{REFERENCES}

[1] C. J. Amick, J. L. Bona and M. E. Schonbek, Decay of solutions of some nonlinear wave-equations, J. Differential Equations, Vol. 81, 1989, pp. 1-49.

[2] M. E. SchonbeK, Decay of parabolic conservation laws, Comm. in Partial Differential Equations, Vol. 7 (1), 1980, pp. 449-473.

[3] M. E. Schonsex, Large time behaviour of the solution to the Navier-Stokes equations, Comm. in Partial Differential Equations, Vol. 11 (7), 1986, pp. 733-763.

[4] M. E. SchonBeK, Lower bounds on the rates of decay of the solutions to the Navier-Stokes equations, J. of the American Math. Soc., Vol. 4, \# 3, July 1991.

[5] M. E. SchonbeK, T. Schonbek and E. SÜLI, Large time behaviour of solutions to the Magneto-Hydrodynamics equation, Preprint.

[6] M. WIEGNER, Decay results for weak solutions of the Navier-Stokes equation on $\mathbb{B}^{n}$, J. London, Math. Soc. Vol. 35 (2), 1987, pp. 303-313.

[7] Z. YULIN and Guo BolING, The periodic boundary-value problems and the initial value problems of the generalised Korteweg-deVries equation, Acta Mathematica Sinica, Vol. 27, 1984, pp. 154-176.

[8] Zhang Linghal, Decay of solutions of higher order multi-dimensional non-linear KdVBurgers system, To appear in the Proc. of the Royal Soc. of Edinburgh.

(Manuscript received January 31, 1994; Revised version received September 7, 1994.)

Vol. $12, \mathrm{n}^{\circ} 4-1995$. 